\title{
Cardiopulmonary Resuscitation Injuries in Children
}

\author{
Kim A. Collins, Christina J. Tatum, and Patrick E. Lantz
}

\section{Contents}

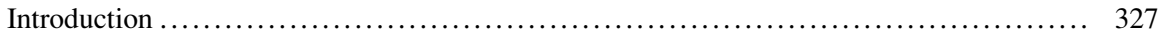

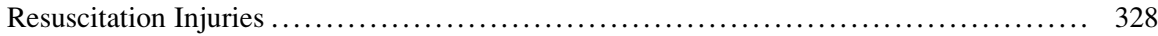

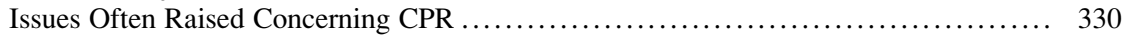

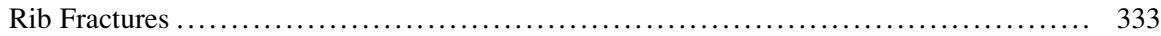

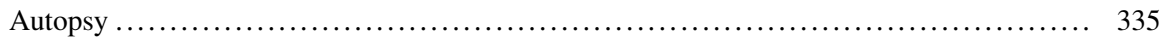

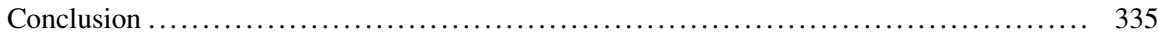

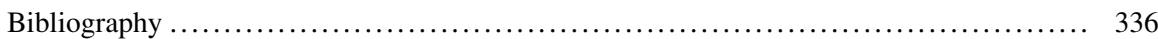

\begin{abstract}
Cardiopulmonary resuscitation (CPR) is an emergency intervention to maintain circulation and breathing in an unresponsive individual suffering from cardiopulmonary arrest. However, CPR is not without its own risks. Injuries secondary to compression and ventilation are well documented in the medical and scientific literature. Most of these injuries are minor, but some can result in significant morbidity and even death. It is important to identify those injuries that could be secondary to CPR versus inflicted traumatic injuries of child maltreatment.
\end{abstract}

\section{Introduction}

Besides medical personnel, members of the general public have been trained to perform CPR. Manual external CPR involves chest compressions at a rate of

\author{
K.A. Collins $(\bowtie)$ \\ Fulton County Medical Examiner's Office, Emory University School of Medicine, Atlanta, GA, USA \\ e-mail: kimcollinsmd@gmail.com \\ C.J. Tatum • P.E. Lantz \\ Wake Forest Baptist Health, Winston Salem, NC, USA \\ e-mail: christinajtatum@yahoo.com; plantz@wakehealth.edu
}


at least 100 per minute for all age groups, at a depth of at least $5 \mathrm{~cm}$ in adults, and at least $1 / 3$ of the depth of the anteroposterior chest diameter in infants and children below the age of puberty. Often a child is brought to the attention of a health-care professional or death investigator, and the etiology of injuries, in particular CPR versus inflicted blunt force trauma, becomes a crucial issue. Perpetrators may claim that injuries were caused by CPR, either by themselves or by emergency personnel. It is important to understand those injuries known to be secondary to CPR in order to better identify and classify inflicted injuries of child maltreatment.

\section{Resuscitation Injuries}

Injuries secondary to CPR are usually pathophysiologically insignificant (Bush et al. 1996; Plunkett 2006; Matshes and Lew 2010a; Ryan et al. 2006; Matshes and Lew 2010b; Reyes et al. 2011). Such injuries may be external and/or internal. It is best to divide the injuries into categories: head and neck, thorax and abdomen, barotrauma, and iatrogenic artifacts. Most CPR injuries involve the head/neck and rarely the thorax and abdomen. These injuries are due to the compressions and to ventilation/ intubation. One should be aware of the resuscitative technique used on children and note if the resuscitator is experienced in this technique (Reyes et al. 2011). The techniques include one-handed compressions ("two finger"), two-handed compressions ("two-thumb-encircling hands"), and abdominal compressions. The emergency medical services (EMS) personnel or physician can easily demonstrate how he/she performed CPR. The investigator can correlate injuries with points of contact during compressions and ventilation. A doll can be used as well as the same type and size of mask. If available, the exact mask used during the resuscitation should be retained. It must be noted that some studies of many resuscitated children report no injuries. In other studies, injuries were noted, but none were significant or abdominal (Matshes and Lew 2010a, b; Price et al. 2000; Feldman and Brewer 1984).

1. Head and Neck: Most CPR-related injuries in children are soft tissue injuries of the head and neck from ventilatory efforts (Kaplan and Fossum 1994). These include facial abrasions (nasal bridge, undersurface of the nose, anterior chin) from the air-bag-valve mask, which are usually symmetrical. As the resuscitator positions his/her hand on the child, fingertip contusions beneath the chin and on the side of the head may be produced. If a mask is not used but instead mouth-to-mouth, one may see scrapes/fingernail scratches over the perinasal area.

Intubation can result in abrasions and/or contusions of the oropharynx, gingiva, buccal mucosa, frenulum, epiglottis, base of the tongue, larynx, and trachea. The lips may be contused or lacerated from the victim's own teeth or by the endotracheal tube (Fig. 14.1). Teeth can also be broken during intubation. The child's oropharynx is more susceptible to damage by forceful digital 
Fig. 14.1 Lip abrasion and contusion from endotracheal tube

Fig. 14.2 Anterior laryngeal submucosal hemorrhage secondary to intubation
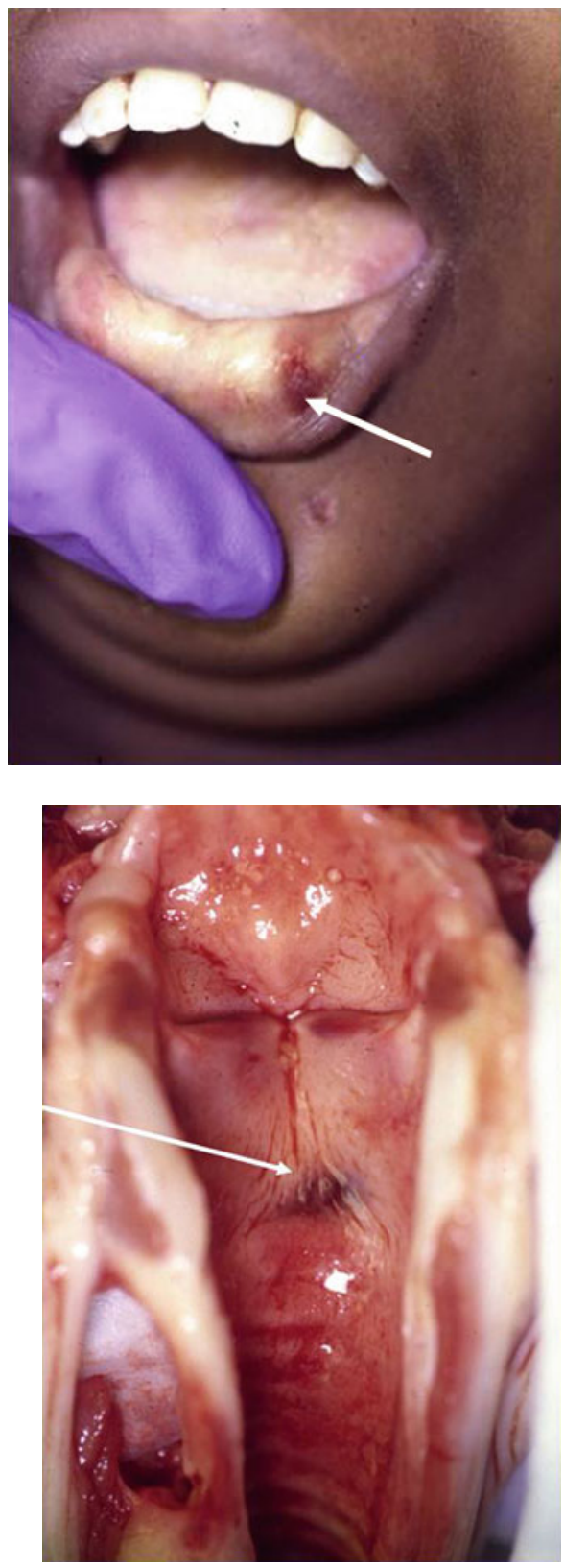

clearing and suction as well as by endoscopic instruments (Figs. 14.2, 14.3). Traumatic mucosal tears and hypopharyngeal perforation have occurred and are not uncommon (Galvis and Kelley 1979).

2. Thorax and Abdomen: Chest compressions can result in midsternal abrasions and/or slight contusions. Epicardial hematoma, pleural petechiae or ecchymosis, 
Fig. 14.3 Pharyngeal edema and soft tissue hemorrhage secondary to finger sweep examination, intubation, and ventilation

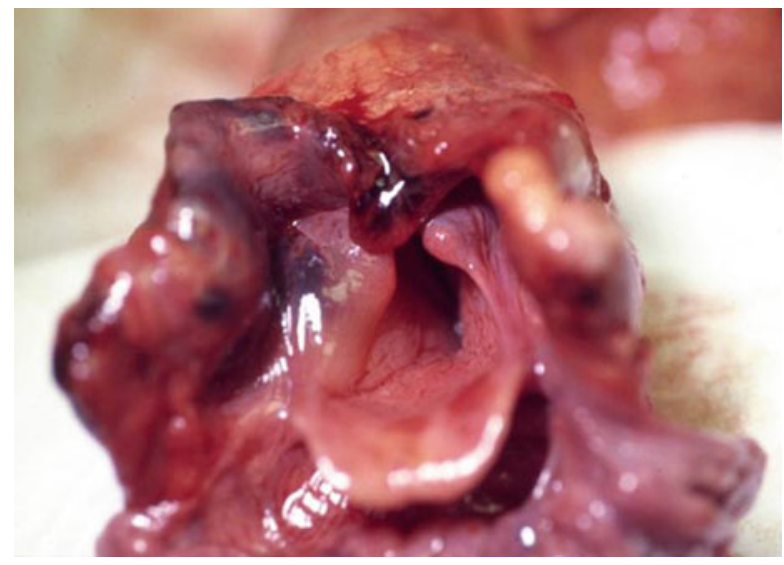

and pulmonary interstitial hemorrhage can also occur (Bush et al. 1996; Matshes and Lew 2010b; Price et al. 2000) (Fig. 14.4a-c). Rib fractures secondary to CPR are rarely reported and are more often the result of inflicted trauma (Bush et al. 1996; Gunther et al. 2000; Spevak et al. 1994; Maguire et al. 2006; Dolinak 2007). Rib fractures are discussed further in the latter portion of this chapter.

Although extremely rare, compressions in children may result in pancreatic hemorrhage, hepatic/splenic contusion or laceration, retroperitoneal hemorrhage, and gastric perforation (Bush et al. 1996; Ryan et al. 2006; Waldman et al. 1984; Custer et al. 1987; Krischer et al. 1987). Note if abdominal compressions were performed (Waldman et al. 1984).

3. Barotrauma: Various forms of CPR-related barotrauma have been described in children (Cullen 2001). These include tympanic membrane injury, pneumothorax, pneumoperitoneum, pneumoscrotum, and air embolism (especially in the premature newborn) (Bush et al. 1996). Less commonly, gastric rupture (usually lesser curvature) due to overdistension during ventilation may result.

4. Artifacts/Iatrogenic Marks Other than from Compression/Ventilation: Artifacts include defibrillator marks over the thorax, venipuncture and intraosseous line access marks, bruising about the neck from attempted vascular access, and adhesive marks from taping the endotracheal tube. Defibrillation can produce subepicardial myofibril disintegration. One can also see cardiac contraction band necrosis and focal hemorrhage with the administration of catecholamines during prolonged resuscitation.

\section{Issues Often Raised Concerning CPR}

- Retinal Hemorrhages (RHs): Previous studies and collaborative research have concluded that CPR alone does not cause retinal hemorrhages in children with normal coagulation and platelet count (Odom et al. 1997). In patients with nontraumatic illnesses and coagulopathies, small, punctate retinal hemorrhages 

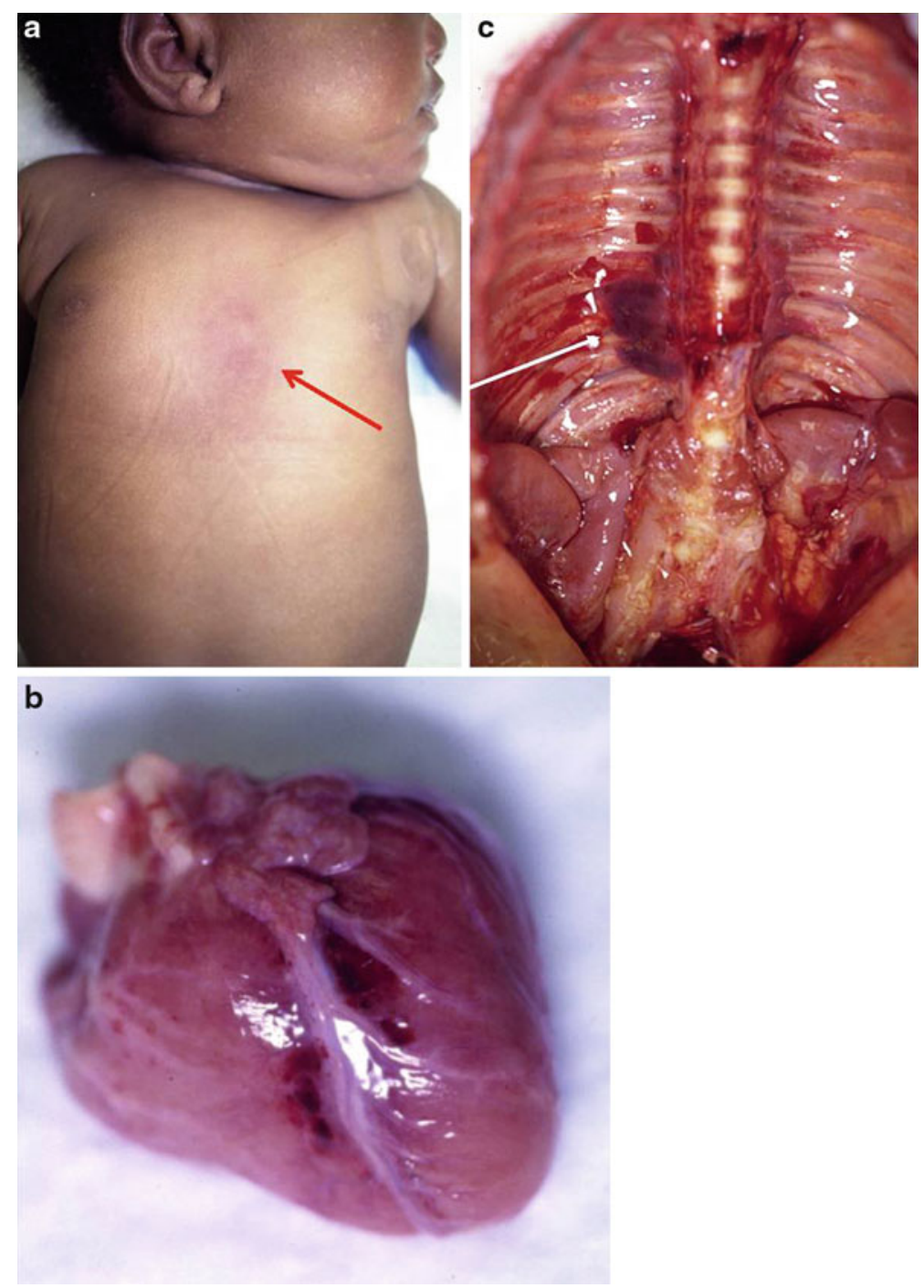

Fig. 14.4 (a) Infant after $45 \mathrm{~min}$ of CPR. Note the anterior chest erythema. (b) Anterior epicardial hemorrhage secondary to chest compressions. (c) After 45 min of chest compressions, no rib fractures were identified. Only slight posterior intercostal hemorrhage

may be present (Odom et al. 1997). Infants found unresponsive invariably undergo resuscitative efforts, often for prolonged periods of time. Forensic pathologists must be aware that retinal hemorrhages can be seen in infants who die suddenly and unexpectedly, following cardiopulmonary resuscitation, and are not specific for abusive head trauma. Infants found unresponsive without evidence of head trauma or natural disease processes that have had CPR, 

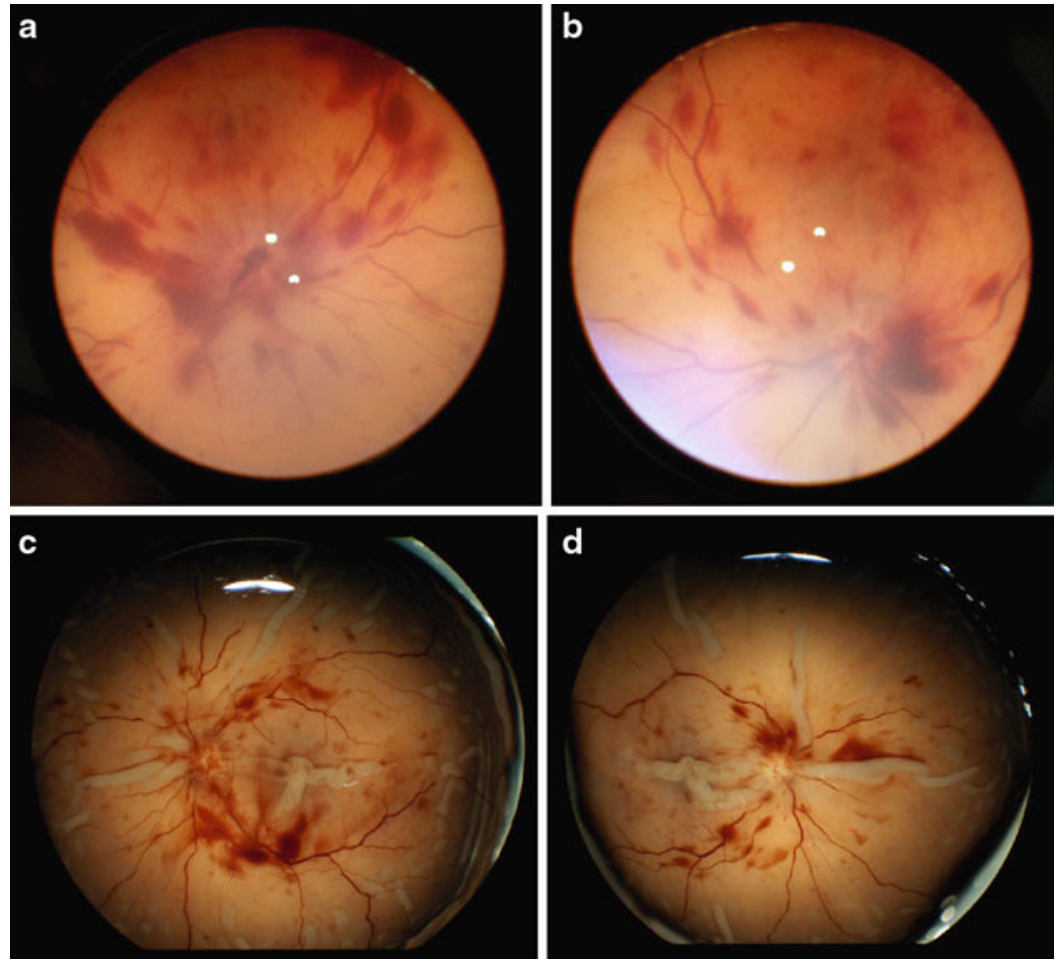

Fig. 14.5 Resuscitation-associated multiple, multi-layered retinal hemorrhages extending past equator and focally abutting ora serrata are likely secondary to hypoxia and reperfusion. (a) Postmortem Indirect Ophthalmoscopy of the left eye. (b) Postmortem Indirect Ophthalmoscopy of the right eye. Fundal images of the left eye (c) and the right eye (d) after enucleation, formalin fixation, and removal of the anterior structures (cornea, iris, ciliary body, and lens). It replicates the clinical view with a direct ophthalmoscope

especially those with restoration of circulation, can have RHs that may be few in number or numerous with extension to the ora serrata (Fig. 14.5a-d) (Tatum et al. 2012).

- Visceral Injuries: Several studies report no visceral injuries to children secondary to CPR (Matshes and Lew 2010a, b; Price et al. 2000; Feldman and Brewer 1984; Cohle et al. 1995). Others report that, if present, these CPR-related injuries are not significant or life-threatening (Matshes and Lew 2010b). Abdominal visceral injury is uncommon (Price et al. 2000); however, if present, note if abdominal compressions were performed (Waldman et al. 1984).

- Rib Fractures: Unlike adults, ribs in children are flexible and more resilient to fracture. In the absence of radiographic evidence of underlying bone disorder, unexplained rib fractures are indicative of abuse. Often rib fractures are associated with other signs of abuse and/or different stages of healing. However, recent studies using axial radiography have shown rib head fractures related to CPR, especially using the two-handed "two thumbs encircling hands" technique 
(Matshes and Lew 2010a; Reyes et al. 2011). Length of compression time does not appear to correlate with an increased chance of rib fractures. Below is a more in-depth discussion of this challenging area in differentiating rib fracture from resuscitation versus inflicted trauma.

\section{Rib Fractures}

Current recommendations by the American Heart Association place emphasis on highquality chest compressions (Field et al. 2010). The two acceptable techniques for chest compressions in infants are the use of either the first and second fingers on one hand or the two-handed technique. Since 2006, the preferred technique for chest compressions is two-handed, with the thumbs generating pressure on the sternum and the second through fifth fingers offering light support on the back (Kattwinkel 2006) (Fig. 14.6).

Reports of CPR in adults suggest fractures of the ribs occur at an incidence of 20-65\%, but such injuries are reportedly rarely seen in children (Ryan et al. 2006; Feldman and Brewer 1984; Krischer et al. 1987; Powner et al. 1984). Prior to the introduction of the preferred two-handed compression technique in 2006, there were only three reported cases of resuscitation-related injuries in children (Maguire et al. 2006). Compared to an adult, the rib cage of a child has greater elasticity and plasticity due to incomplete bone development and therefore may be able to tolerate larger degrees of pressure before a fracture occurs.

Rib fractures found in children with no clinical history of trauma or underlying bone disorders are most often associated with child abuse, accounting for 5-27\% of all skeletal injuries in abused children (Barsness et al. 2003; Platt et al. 2006). Most abusive rib fractures are believed to occur from anteroposterior compression, with the hands wrapped around the infant's chest, produced by excessive squeezing or indirectly generated by shaking. Although abusive compression can cause anterolateral rib fractures, it has been attributed to injuries of the posterior ribs near the costovertebral junction and may involve the rib head, rib neck, and posterior rib arc. The compressive force levers the posterior ribs over the transverse processes of the spine (Kleinman and Schlesinger 1997; Bulloch et al. 2000). Since one-handed CPR is typically performed with the child on a firm surface, this posterior levering should not occur.

When rib fractures are present in an infant who has received CPR, the forensic pathologist must take care to distinguish abusive fractures from those that are related to resuscitative efforts. This is especially true with the two-handed technique, given that significant anterior compression may be produced (Matshes and Lew 2010a; Reyes et al. 2011). Resuscitation-related rib fractures are usually located either anteriorly or anterolaterally and are often multiple, linear, bilateral, or symmetrical (Fig. 14.7). These fractures usually occur near ribs 4 and 5 but have also been reported in ribs 2 through 9 , the clavicle, and the sternochondral junction (Bush et al. 1996; Betz and Liebhardt 1994). If resuscitative efforts are unsuccessful, CPR-induced posterior rib fractures often have minimal associated hemorrhage and may be difficult to identify unless the parietal pleura has been reflected (Fig. 14.8). 
Fig. 14.6 Anterior-posterior radiograph taken while twohanded compression technique was performed in the neonatal intensive care unit (NICU) on a 10-week-old infant. A 2-year-old with a history of upper respiratory infection positive for coronavirus was found unresponsive. He was resuscitated for $40 \mathrm{~min}$ and survived $24 \mathrm{~h}$. No injuries were identified
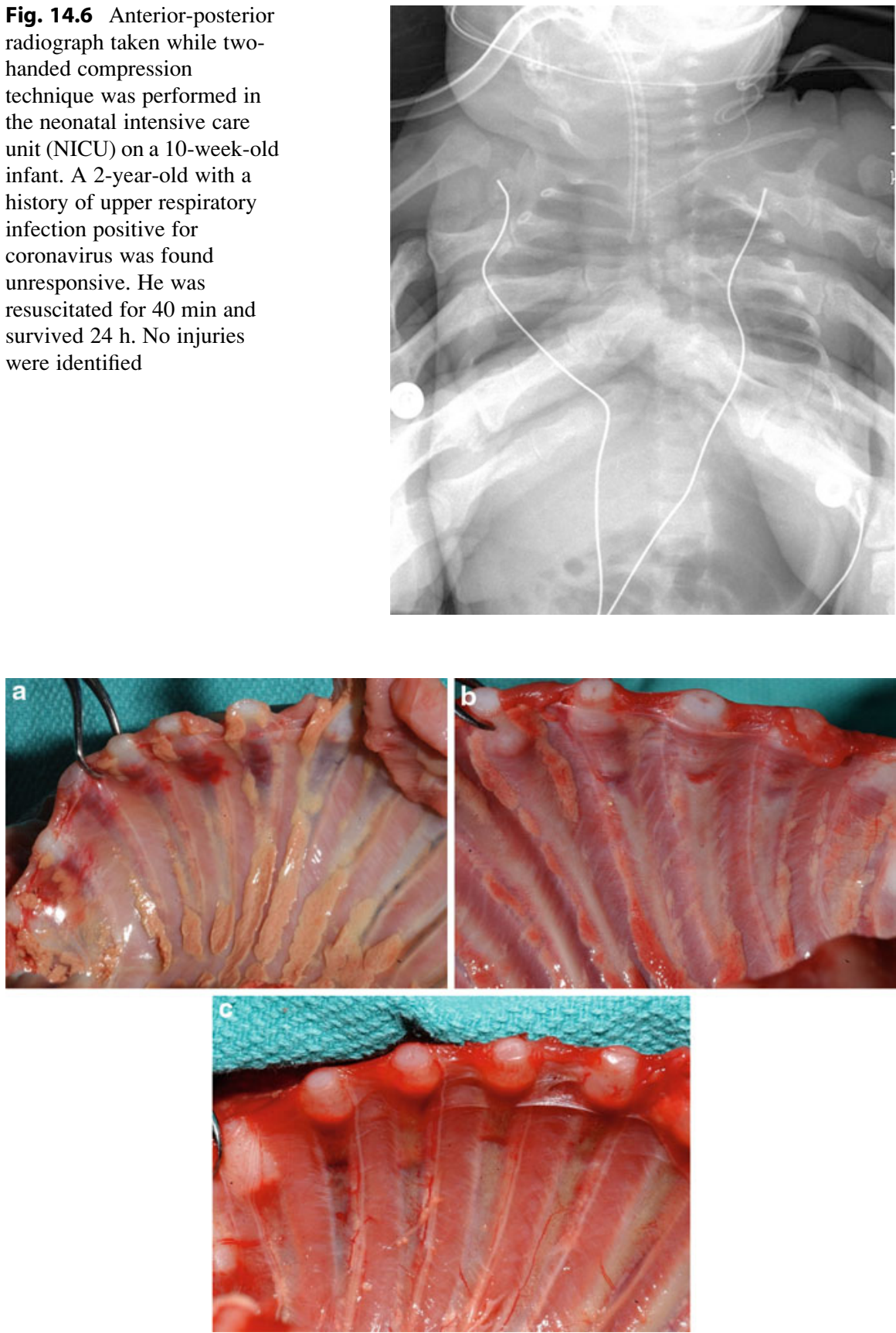

Fig. 14.7 (a) Fractures of left anterior ribs 2-6 with intact pleura in a 10-week-old infant following CPR. (b) Fractures of right anterior ribs 3-5 in a 5-week-old who died suddenly and unexpectedly. (c) Left anterior rib fracture shown with pleura stripped in a 3-month-old after 20 min of CPR 

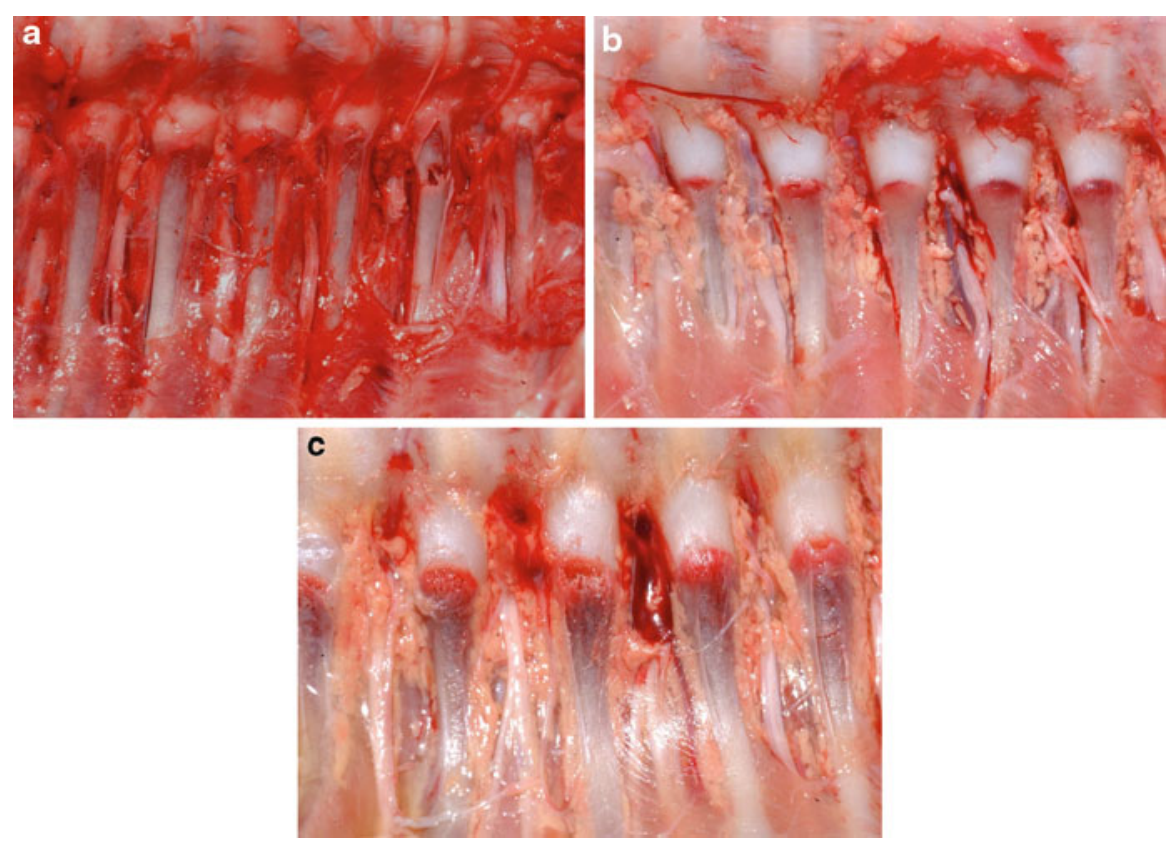

Fig. 14.8 (a) Fracture of the left fourth posterior rib in a 4-month-old who underwent two-handed compression technique. (b) Acute posterior rib head fractures in a 2-day-old who was delivered via emergency $\mathrm{C}$-section following a motor vehicle accident and underwent CPR. (c) Three-monthold infant with fracture of posterior rib/neck after receiving CPR in the NICU

\section{Autopsy}

To detect and confirm the presence of rib fractures at autopsy, a radiologic skeletal survey consisting of full-body, anteroposterior radiographs should always be performed prior to beginning the autopsy. Fresh rib fractures may be missed by radiography, but can be detected at autopsy by stripping the parietal pleura from the thorax and carefully examining each rib by visual inspection and manual palpation. Often the rib fractures will be subtle, bending or buckle fractures, with little or no associated hemorrhage. Microscopic examination of resuscitation-related fractures should show no surrounding tissue reaction with minimal associated extravasated blood histologically (Dolinak 2007; Weber et al. 2009).

\section{Conclusion}

Although usually pathologically insignificant, injuries secondary to CPR do occur and must be distinguished from inflicted injuries of child maltreatment. The investigator must be aware of common injuries of CPR and how these may differ from abusive trauma. Knowledge of the type of resuscitation, mask, and intubation 
instruments used is needed to assess any injuries that may be identified. Doll reenactment may be useful during the investigation. Special attention should be paid to bony fractures and visceral injury as these are not commonly identified as secondary to CPR. Also, any remote injuries should raise suspicion. A complete autopsy preceded by radiologic skeletal survey should be performed.

\section{Bibliography}

Barsness K, Cha E, Bensard D, et al. The positive predictive value of rib fractures as an indicator of non-accidental trauma in children. J Trauma. 2003;54(6):1107-10.

Betz P, Liebhardt E. Rib fractures in children - resuscitation or child abuse? Int J Legal Med. 1994;106(4):215-8.

Bulloch B, Schubert C, Brophy P, et al. Cause and clinical characteristics of rib fractures in infants. Pediatrics. 2000; 105:e48.

Bush CM, Jones SJ, Cohle SD, Johnson H. Pediatric injuries from cardiopulmonary resuscitation. Ann Emerg Med. 1996;28:40-4.

Cohle SD, Hawley DA, Berg KK, Kiesel EL, Pless JE. Homicidal cardiac laceration in children. J Forensic Sci. 1995;40:212-8.

Cullen ML. Pulmonary and respiratory complications of pediatric trauma. Respir Care Clin N Am. 2001;7:59-77.

Custer JR, Polley TZ, Moler F. Gastric perforation following cardiopulmonary resuscitation in a child: report of a case and review of the literature. Pediatr Emerg Care. 1987;3(1):24-7.

Dolinak D. Rib fractures in infants due to cardiopulmonary resuscitation efforts. Am J Forensic Med Pathol. 2007;28:107-10.

Feldman KW, Brewer DK. Child abuse, cardiopulmonary resuscitation, and rib fractures. Pediatrics. 1984;73:339-42.

Field J, Hazinksi M, Sayre M, et al. American Heart Association guidelines for cardiopulmonary resuscitation and emergency cardiovascular care. Circulation. 2010;122:640-56.

Galvis AG, Kelley CF. Hypopharynx perforation during infant's resuscitation. JAMA. 1979;242(14):1526-7.

Gunther WM, Symes SA, Berryman HE. Characteristics of child abuse by anteroposterior manual compression versus cardiopulmonary resuscitation. Am J Forensic Med Pathol. 2000; 21(1):5-10.

Kaplan JA, Fossum RM. Patterns of facial resuscitation injury in infancy. Am J Forensic Med Pathol. 1994;15(3):187-91.

Kattwinkel J. Textbook of neonatal resuscitation. 5th ed. Elk Grove Village: American Academy of Pediatrics; 2006.

Kleinman P, Schlesinger A. Mechanical factors associated with posterior rib fractures: laboratory and case studies. Pediatr Radiol. 1997;27:87-91.

Krischer JP, Fine EG, Davis JH, Nagel EL. Complications of cardiac resuscitation. Chest. 1987;92(2):287-91.

Maguire S, Mann M, John N, Ellaway B, Sibert JR, Kemp AM. Does cardiopulmonary resuscitation cause rib fractures in children? A systematic review. Child Abuse Negl. 2006;30:739-51.

Matshes EW, Lew EO. Two-handed cardiopulmonary resuscitation can cause rib fractures in infants. Am J Forensic Med Pathol. 2010a;31:303-7.

Matshes EW, Lew EO. Do resuscitation-related injuries kill infants and children? Am J Forensic Med Pathol. 2010b;31:178-85.

Odom A, Christ E, Kerr N, Byrd K, Cochran J, Barr F, Bugnitz M, Ring JC, Storgion S, Walling R, Stidham G, Quasney MW. Prevalence of retinal hemorrhages in pediatric patients after inhospital cardiopulmonary resuscitation: a prospective study. Pediatrics. 1997;99:E3. 
Platt M, Spitz D, Spitz W. The abused child and adolescent. In: Spitz W, Spitz D, editors. Medicolegal investigation of death. Springfield: Charles C. Thomas; 2006.

Plunkett J. Resuscitation injuries complicating the interpretation of premortem trauma and natural disease in children. J Forensic Sci. 2006;51:127-30.

Powner D, Holcombe M, Mello L. Cardiopulmonary resuscitation-related injuries. Crit Care Med. 1984;12(1):177-81.

Price EA, Rush LR, Perper JA, Bell MD. Cardiopulmonary resuscitation-related injuries and homicidal blunt abdominal trauma in children. Am J Forensic Med Pathol. 2000;21:307-10.

Reyes JA, Somers GR, Taylor GP, Chiasson DA. Increased incidence of CPR-related rib fractures in infants - is it related to changes in CPR technique? Resuscitation. 2011;82:545-8.

Ryan MP, Young SJ, Wells DL. Do resuscitation attempts in children who die cause injury? Emerg Med J. 2006;20:10-2.

Spevak M, Kleinman P, Belanger P, Primack C, Richmond J. Cardiopulmonary resuscitation and rib fractures in infants. JAMA. 1994;272:617-8.

Tatum CJ, Lantz PE. G20 Retinal hemorrhages associated with non-abusive sudden unexplained deaths in infants. Atlanta: American Academy of Forensic Sciences; 2012.

Waldman PJ, Walters BL, Grunau CFV. Pancreatic injury associated with interposed abdominal compressions in pediatric cardiopulmonary resuscitation. Am J Emerg Med. 1984;2:510-2.

Weber M, Risdon A, Offiah A, et al. Rib fractures identified at post-mortem examination in sudden unexpected deaths in infancy. Forensic Sci Int. 2009;189:75-81. 